\title{
LOS DERECHOS LABORALES EN EL TRATADO DE LIBRE COMERCIO ENTRE COLOMBIA Y ESTADOS UNIDOS
}

\author{
CÉSAR A. RODRÍGUEZ GARAVITO
}

This article briefly presents the main debates on the globalization process and its effects on labor rights, and complements this theoretical framework with a comparative analysis about the inclusion of such rights in recent trade agreements signed by the United States. As a final section, the author presents a specific proposal regarding the inclusion of labor rights in the free trade agreement that is currently under discussion.

\section{Introducción: el problema y las alternativas de solución}

La protección de los derechos laborales está hoy en día en el centro de los debates académicos y políticos sobre la globalización. Al mismo tiempo que la integración económica y el desarrollo orientado hacia las exportaciones se han difundido alrededor del mundo en las últimas tres décadas, gobiernos, académicos y activistas se han ocupado cada vez más de los efectos de tales procesos en las condiciones de trabajo, particularmente en los países en desarrollo.

Como respuesta a las presiones competitivas y a las políticas estatales encaminadas a estimular la integración económica, las empresas transnacionales han intensificado el traslado de sus operaciones con alto componente de mano de obra al Sur global, a través de acuerdos de subcontratación con fábricas que producen para el mercado internacional (Dicken 1998; Gereffi, Korzeniewicz y Korzeniewicz 1994; Gereffi, Spener y Bair 2002). Estas fábricas -conocidas en América Latina como "maquilas"han pasado a simbolizar tanto la forma de producción por subcontratación que las caracteriza como las condiciones laborales precarias presentes en 
muchas de ellas. A medida que ha aumentado la evidencia empírica sobre múltiples formas de violación de los derechos laborales en dichas fábricas desde abusos físicos contra trabajadoras en las plantas productoras de ropa centroamericanas y chinas hasta el uso de mano de obra infantil en las fábricas de balones de fútbol en Bangladesh-, el tema de las condiciones de trabajo en la economía global se ha convertido en objeto de acaloradas disputas tanto académicas como políticas a lo largo de la última década (Fung, O'Rourke y Sabel 2001; Moran 2002; Bonacich y Appelbaum 2002; Ross 1997).

Las manifestaciones de este debate son múltiples. En el ámbito académico, como explicaré en mayor detalle más adelante, la pregunta sobre la conveniencia de proteger los derechos laborales mediante sanciones comerciales en tratados de libre comercio ha creado importantes disensos dentro y fuera de la economía. En el ámbito político, un número importante de organizaciones de derechos humanos, ONG y otras organizaciones de la sociedad civil han creado redes y movimientos sociales transnacionales que abogan por la protección eficaz de los derechos de los trabajadores en la economía global (Keck y Sikkink 1998). En los Estados Unidos, por ejemplo, las campañas contra la explotación laboral global han dado lugar a "la más grande ola de activismo en llegar a las universidades desde los tiempos en que los estudiantes se unieron para liberar a Nelson Mandela a través de un llamado a que se suspendieran las inversiones universitarias en Suráfrica hace más de una década" (Dreier y Appelbaum 1999:12). Trabajando en coordinación con ONG y sindicatos de países en desarrollo, éste y otros movimientos han llevado a numerosas transnacionales a adoptar códigos de conducta empresariales para garantizar el cumplimiento de las normas laborales internacionales en las fábricas con las que éstas contratan (Rodríguez Garavito 2005a, 2005b, 2005c).

En este contexto, uno de los dilemas fundamentales que enfrentan los gobiernos y las organizaciones interestatales es cómo hacer compatible la apertura económica y el goce efectivo de los derechos laborales. En la raíz del dilema se encuentra la "fractura regulatoria" contemporánea entre el derecho nacional y la economía global -esto es, el hecho que "los procesos económicos divergen del modelo para el cual están diseñadas las regulaciones existentes" (Sassen 1998:155). Tal divergencia resulta de dos tendencias de la economía política contemporánea. Por una parte, los procesos económicos operan cada vez más en una escala global, en tanto que las regulaciones estatales operan en la escala del Estado-nación. Por otra parte, la lógica reguladora jerárquica del Estado entra en conflicto con una econo- 
mía organizada cada vez más alrededor de las lógicas de las redes horizontales y los mercados (Jessop 2002).

Las soluciones que se han propuesto a este dilema regulatorio son de cuatro tipos (ver Rodríguez Garavito 2005a). En primer lugar, se ha buscado fortalecer la infraestructura regulatoria internacional, fundamentalmente a través de propuestas de consolidación del poder de supervisión y sanción de la Organización Internacional del Trabajo (OIT), en especial en relación con la violación de los convenios que contienen los llamados "derechos laborales fundamentales" -la prohibición de discriminación en el empleo, la prohibición de formas explotadoras de trabajo infantil, la prohibición de la esclavitud, y la protección de la libertad de asociación sindical y el derecho de negociación colectiva (Elliot y Freeman 2003; Alston 1996). Quienes prefieren esta alternativa ven en ella una forma de establecer un andamiaje regulatorio que opere en la misma escala que los procesos económicos globales y, por tanto, promueva un consenso normativo global en materia laboral similar al que representa la Organización Mundial del Comercio en asuntos comerciales y de propiedad intelectual. En uno y otro caso, se trataría de dotar a la economía mundial del tipo del tipo de "Estado de derecho global" que autores como Sachs (1999) consideran indispensable para gobernar la globalización. En la práctica, aunque aún está lejos de tener los poderes y los recursos para cumplir este papel, la OIT se ha embarcado en programas novedosos - por ejemplo, la supervisión del sector textil de Camboya en el marco del Tratado de Libre Comercio (TLC) entre ese país y los Estados Unidos, sobre el que volveré más adelante-que permiten vislumbrar el potencial de esta alternativa en el largo plazo.

En segundo lugar, en contraposición con el enfoque multilateral y de consenso de la solución anterior, otra alternativa regulatoria ha consistido en la imposición de sanciones unilaterales contra países que violan sistemáticamente los derechos laborales. El caso paradigmático de esta estrategia es el Sistema General de Preferencias de Estados Unidos, en virtud del cual el gobierno de ese país se reserva el derecho de suspender las prerrogativas arancelarias otorgada a otro país cuando concluya que el gobierno de éste vulnera los derechos laborales fundamentales. En la práctica, las sanciones impuestas de esta manera han obedecido más a motivos políticos que al propósito de proteger los derechos laborales, lo que ha suscitado dudas sobre la conveniencia de aproximaciones unilaterales (Alston 1996). Sin embargo, incluso con estas limitaciones, los procesos de evaluación del desempeño de los países que preceden a las certificaciones anuales necesarias para renovar los beneficios del SGP han servido como escenario para la de- 
nuncia y la discusión pública de las prácticas más graves de violación de los derechos humanos de los trabajadores en países como Guatemala y El Salvador.

En tercer lugar, la ausencia de regulación estatal e interestatal eficaz ha llevado al surgimiento de formas privadas de regulación, esto es, códigos de conducta empresariales que estipulan los derechos laborales que deben respetar los contratistas de las empresas transnacionales que los adoptan. Estas formas de "derecho suave" o "gobernanza" han proliferado en la última década, hasta el punto que un estudio reciente del Banco Mundial (2003) calcula que hoy existen 1.000 códigos de conducta que regulan las condiciones laborales y ambientales de las principales empresas transnacionales. La conveniencia y los efectos prácticos de los códigos de conducta y de los mecanismos de supervisión que han sido creados para implementarlos son objeto de una discusión que apenas comienza (ver Rodríguez Garavito 2005a, 2005b, 2005c).

En cuarto lugar, las soluciones más relevantes para el tema específico de este artículo son las relacionadas con la incorporación de cláusulas sobre derechos laborales en tratados de libre comercio. Como explicaré en páginas posteriores, las propuestas y las experiencias existentes son variadas, y van desde la incorporación de una lista exhaustiva de derechos laborales y de sanciones comerciales en los textos constitutivos de organizaciones multilaterales como la OMC hasta la inclusión de declaraciones de principios sin efecto coercitivo en tratados bilaterales de libre comercio. El resto de este escrito se concentra en este ámbito, específicamente en las aproximaciones alternativas a la protección de los derechos laborales en tratados bilaterales de libre comercio como el que Colombia comenzó a negociar con los Estados Unidos en mayo de 2004.

A pesar de que en la siguiente sección haré alusión a los debates teóricos sobre la pregunta acerca de la conveniencia de introducir cláusulas laborales en tratados de libre comercio, en este escrito parto de la base que, para los efectos prácticos de la negociación del TLC entre Colombia y Estados Unidos, la respuesta a esa pregunta está dada de antemano por la legislación de uno y otro país. En efecto, dado que "la promoción de los derechos laborales" es una de las condiciones establecidas por el Congreso de Estados Unidos en el Bipartisan Trade Promotion Authority (TPA) -la ley firmada por el Presidente Bush el 6 de agosto de 2002 que otorgó facultades especiales de negociación comercial ("fast track" authority) al gobierno estadounidense y en virtud de la cual éste firmaría el TLC con el gobierno colombiano-, la 
inclusión de derechos laborales y de mecanismos de protección es un requisito ineludible para Estados Unidos en las negociaciones. Así lo muestran no sólo los debates en el Congreso que llevaron a la expedición de la ley TPA, sino el hecho que todos los TLC que el gobierno estadounidense ha firmado con base en dicha ley -con Chile y Singapur en 2003 y con Marruecos en 2004, así como el tratado pendiente de ratificación legislativa con los países del CAFTA (Costa Rica, Guatemala, Honduras, El Salvador y Panamá) contienen cláusulas referentes a la protección de derechos laborales. Aunque no existe una ley equivalente en el ordenamiento jurídico colombiano, ${ }^{1}$ la protección al trabajo dentro de una economía social de mercado establecida en la Constitución, tal como ha sido interpretada por la jurisprudencia de la Corte Constitucional, lleva a la misma conclusión en relación con la necesidad legal de estipular expresamente en el TLC los derechos laborales y los mecanismos de protección que los dos gobiernos se comprometen a impulsar. Por estas razones, en suma, la discusión práctica en nuestro medio no es sobre si se debe o no incluir una cláusula laboral en el TLC, sino sobre cuáles deben ser los detalles de dicha cláusula.

En vista de esto, sorprende la poca atención que se ha prestado hasta el momento al tema en el país, así como la resistencia de prominentes analistas económicos a cualquier alusión a la protección de derechos humanos en el contexto de la negociación del TLC (Reina 2004). Dado el hecho de las negociaciones del TLC, es indispensable reflexionar y debatir las características de la cláusula laboral de dicho tratado con base en un análisis juicioso del contexto colombiano, de las experiencias comparadas y de las condiciones económicas, jurídicas y políticas que rodean al TLC.

Con el fin de contribuir a esta tarea, en este artículo hago un balance de los casos de los tratados de libre comercio que han incorporado cláusulas laborales e indago el tipo de cláusula que mejor se ajustaría al propósito de proteger eficazmente los derechos laborales en Colombia en el marco del TLC. Con base en este análisis, sostengo que una cláusula que incluya los derechos laborales reconocidos internacionalmente y que establezca un organismo independiente de revisión de quejas e imposición de sanciones no comerciales es el mecanismo adecuado para simultáneamente promover la protección de los derechos laborales y evitar los riesgos de proteccionismo y

\footnotetext{
${ }^{1}$ Fue presentado, sin embargo, un proyecto de ley (No. 217/02 de abril 14 de 2004) que estipula que "los acuerdos de comercio no pueden vulnerar o poner en peligro derechos fundamentales reconocidos en la Constitución y los tratados internacionales".
} 
de efectos secundarios contraproducentes que los críticos de las cláusulas laborales señalan como razón para oponerse a ellas. Este tipo de cláusula, como se verá a continuación, surge del cúmulo de experimentos y lecciones de mecanismos similares en diversos tratados de libre comercio recientes.

Para presentar este análisis y la evidencia empírica que lo respalda, divido el resto del artículo en tres partes. En la primera, aludo brevemente a los principales debates en relación con la creación de vínculos entre la globalización y los derechos laborales a través de cláusulas sociales en tratados de libre comercio. En la segunda, comparo los modelos de cláusulas laborales que han sido utilizados en el NAFTA y en los tratados bilaterales y multilaterales recientes firmados por Estados Unidos. En la tercera parte, con base en el balance del análisis comparado, concluyo formulando una propuesta concreta en relación con el contenido de la cláusula laboral en el TLC entre Colombia y Estados Unidos.

\section{El debate sobre las cláusulas laborales}

\subsection{La discusión teórica}

Un síntoma de la importancia económica y política de las cláusulas laborales es la profundidad de las divisiones dentro y fuera de la academia en relación con la conveniencia de utilizar los tratados de libre comercio como mecanismos para proteger los derechos laborales. Dentro de los círculos académicos, el tema ha provocado confrontaciones públicas inusuales entre algunos de los economistas más prominentes de Estados Unidos y otros países, que revelan la ausencia de un consenso teórico al respecto. En el seno de la economía, a diferencia de lo que sucede en otras disciplinas o en la opinión pública, el disenso no se refiere a la bondad del libre comercio -que los economistas dan por sentada-, sino a la conveniencia de vincular la protección de los trabajadores con la promoción del comercio internacional.

El debate entre destacados economistas en relación con el nexo entre derechos laborales y comercio se ha dado entre dos grandes posiciones. De un lado, autores como Jagdish Bhagwati -quien desde la Universidad de Columbia se ha convertido en uno de los defensores más pugnaces de la globalización sin condiciones (Bhagwati 2004) - han criticado consistentemente durante años las cláusulas laborales y abogado por una agenda comercial que no incluya los derechos laborales. Conforme con este punto de vista, Bhagwati y un grupo prominente de economistas y científicos sociales de diversos países se han organizado para oponerse al movimiento estudiantil estadounidense que reclama protecciones laborales para los tra- 
bajadores del Tercer Mundo a través de tratados códigos de conducta exigentes, así como a las propuestas de sindicatos y otras organizaciones de introducir cláusulas laborales en tratados de libre comercio como el de la OMC (Bhagwati 2001). Los argumentos centrales esgrimidos por quienes defienden esta posición son dos. De un lado, sostienen que las cláusulas laborales son formas veladas de proteccionismo mediante las que los gobiernos y sindicatos de los países desarrollados se reservan el derecho de imponer sus estándares laborales a los países desarrollados con los que comercian, a pesar de que la realidad económica y social de estos es muy diferente. De esta manera, los primeros privan a los segundos de la ventaja que tienen en el comercio internacional, esto es, los bajos costos laborales. De otro lado, afirman que, incluso cuando los motivos subyacentes a las cláusulas laborales son altruistas, éstas son contraproducentes. Dado que, desde este punto de vista, la mejor manera de mejorar los estándares laborales es mediante un crecimiento económico sostenido que se logra a través de un comercio libre, saturar los tratados y las instituciones de libre comercio con cargas relativas a derechos laborales termina creando un obstáculo para el funcionamiento de dichas instituciones y, por tanto, para el crecimiento económico. Por esto, desde esta perspectiva, los derechos laborales deben ser promovidos mediante mecanismos no coercitivos de cooperación internacional como los encarnados actualmente por la OIT o por formas de autorregulación como los códigos de conducta (Bhagwati 1999, 2001).

Del otro lado, un número importante de economistas prominentes y científicos sociales de diferentes disciplinas ha defendido la utilización de cláusulas laborales. La manifestación más visible y desarrollada de esta posición son los trabajos de Richard Freeman, profesor de econonomía en Harvard que, en colaboración con Kimberly Elliot del Instituto de Economía Internacional, ha liderado el esfuerzo por hacer compatibles los imperativos del libre comercio y los de la protección de los derechos laborales (Elliot y Freeman 2003). En explícita confrontación con Bhagwati y los proponentes de las tesis contra el nexo entre derechos laborales y libre comercio, Freeman y los demás miembros de esta red académica han respaldado el movimiento global contra la explotación en las maquilas y el uso de cláusulas laborales en tratados de libre comercio. Los dos argumentos principales que se ofrecen para sustentar esta posición son respuestas a las críticas hechas desde el bando opuesto. Primero, para quienes defienden esta posición las cláusulas laborales no han surgido de motivaciones proteccionistas ni han sido utilizadas con este fin. Como Elliot y Freeman (2003, cap. 4) lo han mostrado en su análisis del uso de las cláusulas laborales por parte de gobiernos y ONG, la gran mayoría de los casos no ha buscado cerrar el comercio a los países en 
desarrollo sino presionar a los gobiernos de estos últimos para que apliquen los estándares laborales mínimos en casos de violaciones abiertas y sistemáticas. Segundo, desde este punto de vista el libre comercio y los derechos laborales no son fines en conflicto, sino componentes esenciales de un mismo andamiaje económico y político capaz de producir un crecimiento económico que beneficie a la mayor parte de la población mundial. La protección eficaz de los derechos laborales, de acuerdo con numerosos estudios hechos desde esta perspectiva, genera las condiciones políticas (p.ej., democracia dentro y fuera del lugar de trabajo) y sociales (p.ej., disminución de la pobreza y la desigualdad) esenciales para que los países se inserten exitosamente en la economía mundial y para que el crecimiento generado de esta manera sea equitativo y socialmente sostenible (MacEwan 2001; Baker, Epstein y Pollin 1998).

Esta posición, que está en la base de la propuesta hecha en este artículo a favor de una cláusula laboral adecuada en el TLC entre Colombia y Estados Unidos, aduce una razón adicional que generalmente es soslayada por quienes critican este tipo de mecanismo de protección de derechos laborales. Dado que los tratados de libre comercio -incluyendo la OMC a través del acuerdo TRIPS (Trade-Related Aspects of Intellectual Property Rights) han incorporado normas estrictas de protección de derechos de propiedad intelectual, la inclusión de cláusulas laborales en dichos tratados es una exigencia mínima de coherencia económica y política. Si el argumento contra las cláusulas laborales se basa en la necesidad de mantener cualquier asunto distinto del libre comercio por fuera de los TLC, ipor qué se han incorporado regulaciones detalladas de los derechos de propiedad intelectual? La inconsistencia de esta posición es resaltada por Elliot y Freeman (2003:24) al comentar críticamente la protección fuerte de los derechos de propiedad intelectual mediante el acuerdo TRIPS y la oposición a una protección similar para los derechos laborales:

Los proponentes del TRIPS querían reemplazar la colcha de retazos de leyes y prácticas nacionales inadecuadas sobre la propiedad intelectual por una protección de alto nivel basada en estándares internacionales, y aplicar las nuevas reglas mediante sanciones comerciales. Si se reemplaza el término "propiedad intelectual" por "derechos laborales" en la frase anterior, se tiene el argumento a favor de las cláusulas laborales: reemplazar la colcha de retazos de leyes y prácticas nacionales inadecuadas sobre los derechos laborales por una protección de alto nivel basada en estándares internacionales, y aplicar las nuevas reglas mediante sanciones comerciales. 
Este argumento es contundente en el caso colombiano, dado que el gobierno nacional -a diferencia de otros países como Brasil o Suráfrica-ha adoptado precisamente este tipo de protección estricta de los derechos de propiedad intelectual bajo la amenaza de sanciones comerciales. El caso emblemático de esta tendencia fue la expedición del Decreto 2085/2002, mediante el cual se adoptó un régimen estricto de protección de la propiedad intelectual sobre medicamentos para cumplir las condiciones establecidas por el gobierno estadounidense para renovar las preferencias arancelarias vinculadas a la lucha contra el narcotráfico, en detrimento de la producción de medicamentos genéricos accesibles a la mayor parte de la población colombiana.

\subsection{La discusión práctica}

Independientemente de la posición que se tenga en relación con este debate teórico, la discusión práctica está determinada por las condiciones jurídicas, económicas y políticas que a las que se sujeta cada tratado de libre comercio. En el del TLC entre Colombia y Estados Unidos, como lo mostré en la sección introductoria, estas limitaciones -fundamentalmente derivadas de los requisitos de la ley TPA del Congreso norteamericano y de la Constitución colombiana- hacen que, para efectos prácticos, el debate teórico sea resuelto la favor de la inclusión de cláusulas laborales en el TLC.

Esta razón jurídica está reforzada por motivos económicos, sociales y políticos. En cuanto a las razones económicas y sociales, la experiencia de los diez primeros años del NAFTA, que ha sido objeto de numerosos balances recientes, muestra que aunque los tratados de libre comercio promueven el aumento de la productividad, ésta no se traduce automáticamente en más y mejores empleos y en un crecimiento equitativo. Como lo concluye el estudio exhaustivo del Carnegie Endowment for International Peace sobre la primera década del NAFTA, "si [los] aumentos en la productividad han de compartirse con los trabajadores a través del incremento de los salarios, deben fortalecerse las instituciones y las políticas públicas que afectan el resultado de los salarios. Los países con leyes e instituciones débiles en torno de la libertad de asociación y la negociación colectiva deben abordar estos problemas junto con la liberalización del comercio" (Audley, Papademetriou, Polaski y Vaughan 2003:36). En otras palabras, el nexo entre el libre comercio y un desarrollo económico y social sostenible y equitativo depende en parte de la garantía de los derechos laborales. Estos, por lo tanto, deben ser parte integral de una política económica y social colombiana abierta al mercado internacional. 
Las razones políticas a favor de una cláusula laboral adecuada en el TLC surgen del contexto específico colombiano y están íntimamente ligadas a las anteriores consideraciones económicas y sociales. Si en general es aconsejable tener disposiciones laborales garantistas en los TLC, lo es mucho más en el caso colombiano, caracterizado por algunas de las violaciones más graves de los derechos de los trabajadores en el mundo. Basta mencionar la violación sistemática y grave a dos de los cuatro derechos laborales fundamentales reconocidos mediante las ocho convenciones universales de la OIT. Por un lado, la prohibición de las peores formas de trabajo infantil -posiblemente el derecho que ha generado más consenso en la comunidad internacional- es violado cotidianamente mediante la participación de los menores en diferentes bandos y tareas del conflicto armado. De otro lado, Colombia tiene el triste récord de ser el país con el mayor número de asesinatos de sindicalistas y líderes sindicales. De acuerdo con el reciente concepto presentado por el International Labor Rights Fund ante el Congreso de Estados Unidos a propósito de la iniciación de las negociaciones del TLC, en el período 1991-2002 fueron asesinados 1.925 líderes sindicales. Durante 2002, 90 sindicalistas fueron asesinados, 295 fueron amenazados de muerte, 20 sufrieron ataques físicos y 6 fueron secuestrados (International Labor Rights Fund 2004). Por esta razón, Colombia es el único país latinoamericano y uno de los pocos del mundo que ha sido objeto de una investigación a profundidad de la OIT, reservada para las violaciones más graves de los derechos laborales. Igualmente, semejantes violaciones de los derechos de asociación sindical y negociación colectiva ha dado lugar al mayor número de observaciones condenatorias (13 hasta finales de 2001) del Comité para la Aplicación de las Convenciones y Recomendaciones de la OIT (Elliot 2003:12).

En este contexto, por lo tanto, las razones políticas y morales a favor de una cláusula laboral eficaz tienen que ver con la protección de derechos humanos fundamentales como la vida y la integridad física. A diferencia de lo que ha sucedido en otros tratados de libre comercio celebrados por Estados Unidos con países como México o Chile, donde la gravedad de los problemas laborales son un pálido reflejo de la del violento contexto colombiano, la oposición política a un TLC sin derechos laborales exigibles, tanto en Estados Unidos como en Colombia, es una razón de peso que debe ser tomada en cuenta seriamente al momento de redactar el tratado. Como se verá más adelante, las dificultades actuales del CAFTA en el Congreso de los Estados Unidos y la oscilación del péndulo político en ese país a favor de la protección de derechos laborales en los TLC, ofrecen advertencias que no pueden ser ignoradas ni siquiera por los empresarios y asesores económicos cuya única prioridad es la aprobación del tratado. 


\section{Las experiencias comparadas}

Los precedentes de tratados celebrados en los últimos diez años proporcionan lecciones positivas y negativas de gran valor para el diseño de la cláusula laboral del TLC entre Colombia y Estados Unidos. En esta sección, examino someramente los principales tratados de libre comercio celebrados por Estados Unidos en los últimos años que contienen estipulaciones sobre derechos laborales.

Para hacer inteligible y comparable el análisis de los textos complejos de estos acuerdos, conviene organizar el estudio de las cláusulas laborales alrededor de dos conjuntos de preguntas. El primer interrogante es: icuál es el alcance de los derechos consagrados en el tratado? En el transfondo de esta pregunta está una amplia discusión, que no es del caso retomar acá, sobre cuáles deben ser los derechos mínimos aplicables en los acuerdos de libre comercio. Las posiciones en esa discusión van desde las de aquellos que se oponen a la imposición de estándares uniformes a países con niveles de desarrollo muy distintos hasta las de quienes sostienen que una lista específica de derechos (por ejemplo, los cuatro derechos fundamentales de la OIT) deben ser aplicados universalmente (ver, en general, Garay 2002). En esta sección dejo de lado este importante debate para concentrarme en la comparación del alcance de las cláusulas existentes. Como lo ha sostenido Weiss (2003:708), con el fin de comparar rigurosamente los compromisos adquiridos por los países en relación con los derechos protegidos por los tratados de libre comercio, es necesario dividir esta pregunta en cuatro puntos: 1) la lista de derechos incluida en la cláusula; 2) las fuentes de las que provienen esos derechos, que determinan si los países se comprometen sólo a cumplir con sus leyes laborales internas o si también se comprometen a que éstas sean acordes con los estándares laborales internacionales de la OIT; 3) la seriedad del compromiso adquirido, evaluada con base en los términos del tratado y en el tipo de infracción que puede dar lugar a los mecanismos de consulta o sanción; y 4) las obligaciones procesales -acceso a la justicia, debido proceso, etc.- que adquieren los países firmantes para hacer exigibles en sus jurisdicciones los derechos consagrados en la cláusula.

El segundo grupo de preguntas tiene que ver con el procedimiento, los órganos y los mecanismos de control y sanción. ¿Quién está facultado para presentar quejas por violaciones de los derechos incluidos en la cláusula? ¿Quién investiga y decide los casos? ¿Qué tipo de consultas o sanciones están establecidas en caso de infracción de la cláusula? En el trasfondo de estas preguntas prácticas también se encuentran debates importantes sobre 
la conveniencia de diversos tipos de procedimiento y sanción. Por ejemplo, existe un diálogo interesante entre los especialistas en relación con la conveniencia de dejar el procedimiento en manos de los países firmantes, de un órgano internacional como la OIT o de un organismo independiente establecido específicamente para solucionar las controversias surgidas del tratado (Alston 1996; Diamond 1996; Elliot 2003). Igualmente, uno de los debates más relevantes gira alrededor del tipo de sanciones que debe ser impuesta a un país renuente a cumplir los compromisos adquiridos en virtud de la cláusula laboral. En general, tras una preferencia inicial por duras sanciones comerciales consistentes en imposición de aranceles a los países infractores, las organizaciones de defensa de derechos laborales y los analistas independientes han convergido gradualmente hacia una posición que favorece la imposición de multas o, en el peor de los casos, sanciones comerciales sólo a los sectores productivos involucrados en el caso. Al no imponerse sanciones comerciales generales al país, se evita el riesgo del uso proteccionista y los efectos contraproducentes de éstas sobre la población en general (Elliot y Freeman 2003; Human Rights Watch 2002).

Veamos entonces brevemente las experiencias comparadas con base en estos dos grupos de criterios. En lo que sigue, aplico en detalle estos dos ejes al análisis del precedente fundamental -el acuerdo laboral paralelo al NAFTA-, para luego, contra este telón de fondo, esbozar con trazos más generales las innovaciones introducidas por tratados posteriores.

\subsection{El NAFTA y el Acuerdo Laboral de Cooperación Laboral de América del Norte}

El primer antecedente de cláusulas laborales en tratados de libre comercio en el hemisferio es el Acuerdo de Cooperación Laboral de América del Norte (ACLAN). El hecho de que el ACLAN sea un acuerdo paralelo al NAFTA y no parte del texto de éste se explica por las circunstancias políticas que le dieron origen. Tras la firma de NAFTA en 1992, las críticas de numerosos analistas, movimientos sociales y políticos, sindicatos y organizaciones de la sociedad civil en los tres países del NAFTA contra la concentración exclusiva del tratado en la protección del libre comercio y los derechos de propiedad llevaron a la firma posterior de dos acuerdos separados destinados a proteger los derechos laborales (ACLAN) y el medio ambiente (ACAAN).

A pesar de la premura con que se suscribió el ACLAN y la inexistencia de precedentes al momento de su firma, el acuerdo contiene innovaciones 
que abrieron la puerta a la experimentación con cláusulas laborales que continúa hoy en otros tratados. Veamos entonces las características del modelo ACLAN en relación con los dos ejes de comparación que fueron delineadas anteriormente.

\subsubsection{El alcance de los derechos}

Con base en los cuatro criterios propuestos para evaluar el alcance de los derechos (lista de derechos, fuentes, seriedad del compromiso y obligaciones procesales), los aspectos principales del ACLAN pueden ser resumidos de la siguiente manera. En relación con los derechos garantizados, el acuerdo incluye una lista de 11 principios que las partes se comprometen a impulsar:

1. Libertad de asociación y derecho de asociación sindical;

2. Derecho de negociación colectiva;

3. Derecho a la huelga;

4. Prohibición de trabajo forzado;

5. Protecciones laborales para niños y jóvenes;

6. Garantía de derechos laborales mínimos;

7. Eliminación de la discriminación en el trabajo;

8. Igual remuneración para mujeres y hombres;

9. Prevención de lesiones y enfermedades profesionales;

10. Compensación en caso de lesiones o enfermedades profesionales; y

11. Protección para trabajadores migrantes.

Lo notable de esta lista es que incluye y va más allá de los cuatro derechos laborales fundamentales reconocidos por la OIT, (prohibición del trabajo forzado, prohibición de la discriminación, derecho de asociación sindical y negociación colectiva, y eliminación de formas explotadoras de trabajo infantil). Entre las garantías adicionales que merecen ser resaltadas se encuentran el pago de salario mínimo (como parte del principio 6) y la expresa protección del derecho a la huelga. Esto ha hecho que numerosos analistas y organizaciones de defensa de los derechos humanos hayan acogido la lista de principios del ACLAN como un modelo adecuado para futuros tratados de libre comercio (Hufbauer y Schott 2002; Human Rights Watch 2001; Weiss 2003).

En cuanto a las fuentes de estos derechos, el compromiso central de México, Estados Unidos y Canadá en virtud del ACLAN es "promover el cumplimiento y la aplicación eficaz de su respectivo ordenamiento laboral 
[interno] a través de acciones gubernamentales apropiadas". Por lo tanto, la fuente central es el derecho nacional, no los convenios universalmente adoptados a través de la OIT. Como lo ha anotado Weiss (2003), el ACLAN equivale en efecto a la creación de una nueva obligación internacional consistente en aplicar el derecho nacional.

Las ventajas y limitaciones de esta solución son fácilmente discernibles. Por una parte, permite alcanzar una solución de compromiso en casos en los que un país firmante, a pesar de no haber suscrito la mayor parte de los convenios de la OIT, tenga una legislación laboral interna que protege de manera relativamente eficaz los derechos básicos. Estados Unidos es el ejemplo paradigmático de esta situación. La falta de inclusión del derecho laboral internacional como fuente de obligaciones tampoco constituye un problema serio cuando el país firmante tiene un derecho laboral interno que incorpora los convenios de la OIT, a pesar de que su aplicación sea débil. Si existe una legislación interna garantista y estable, y una obligación (en virtud de tratados como el ACLAN) de aplicarla estrictamente, una cláusula cláusula laboral de este tipo puede crear un mecanismo de presión adicional sobre el Estado.

Por otra parte, sin embargo, la falta de incorporación de los derechos laborales internacionales en los tratados de libre comercio tiene dos desventajas importantes. En primer lugar, erosiona el consenso emergente alrededor del mundo en relación con un derecho laboral global surgido de las convenciones de la OIT, y lo reemplaza por sistemas unilaterales o bilaterales (Alston 1996). En segundo lugar, en la práctica la tentación de los gobiernos de reformar el contenido o la interpretación de su derecho interno cuando se alegue que éste no se cumple resulta demasiado fuerte cuando la única obligación consiste en respetar el derecho nacional independientemente de su consonancia con las normas de la OIT. Es esto precisamente lo que ha sucedido en la implementación del ACLAN. En efecto, las Oficinas Administrativas Nacionales (OAN) encargadas de estudiar las quejas han tendido a entender la obligación de los estados miembros en términos laxos, hasta el punto de aceptar invariablemente la interpretación particular de los gobiernos sobre sus normas internas y la posibilidad de reformarlas, independientemente de su concordancia con los derechos laborales internacionales de la OIT. Esto fue evidente, por ejemplo, en el caso Duro Bag, en el que la OAN de Estados Unidos en 2001 rechazó la acusación de violación del derecho de asociación sindical contra México relacionada con la legislación de este país que impide la realización de elecciones de sindicatos independientes mediante voto secreto de los trabajadores. Para la OAN estadouni- 
dense, la legislación laboral y la interpretación de la misma del gobierno mexicano -que permite la presencia de los empleadores en las elecciones sindicales y, así, continúa la tradición de cooptación del sindicalismo mexicano- no son incompatibles con la obligación de México de aplicar su derecho interno.

La lección del ACLAN muestra, en síntesis, que en relación con las fuentes de los derechos estipulados en las cláusulas laborales es aconsejable incluir tanto el derecho interno como los convenios de la OIT ratificados por cada uno de los países firmantes. Esta solución contribuye al surgimiento de una legislación laboral internacional y evita la modificación o interpretación caprichosa de la legislación por parte de un país acusado de violaciones de dichos derechos. Al mismo tiempo, deja un margen de flexibilidad para que no se imponga de entrada un conjunto específico de convenciones de la OIT que los estados firmantes (por ejemplo, Estados Unidos) pueden no haber ratificado.

En relación con la seriedad del compromiso adquirido por las partes en virtud del acuerdo en relación con la protección de estos derechos, el ACLAN establece tres niveles de exigibilidad.

1. El primer nivel comprende los derechos laborales colectivos (principios 1, 2 y 3), que son objeto del compromiso y la protección más débil. Su violación acarrea sólo la supervisión de las OAN y, a lo sumo, consultas entre los ministerios de trabajo de los tres países, cuyo funcionamiento es explicado en la siguiente sección. La infracción de estos derechos, por tanto, no puede dar lugar a sanciones ni evaluaciones por parte de comités de expertos independientes.

2. El segundo nivel incluye los derechos relacionados con el trabajo forzado, la discriminación en el lugar de trabajo, igualdad de remuneración entre géneros, compensación en caso de enfermedad o lesión, y la protección de los trabajadores migrantes. En caso de violación de estos derechos, conforme con el procedimiento descrito más adelante, los mecanismos previstos por el ACLAN son la supervisión de las OAN, consultas ministeriales y la evaluación de un comité de expertos. Sin embargo, a los derechos incluidos en este nivel no se aplican los mecanismos más exigentes del sistema en caso de falta de cooperación del país transgresor, esto es, el arbitraje y la imposición de sanciones. 
3. Finalmente, en el tercer nivel se encuentran los principios relativos al trabajo infantil, salario mínimo y seguridad industrial. A estos se aplican todos los mecanismos de supervisión y sanción del tratado.

Esta división entre niveles de protección ha sido ampliamente criticada en la bibliografía por establecer una discriminación injustificada entre principios igualmente importantes, por complicar aún más el sistema de protección de los derechos incluidos en el ACLAN, y por haber dificultado en la práctica la garantía de derechos violados repetidamente en México y Estados Unidos, especialmente el de asociación sindical y huelga (Elliot 2003; Elliot y Freeman 2003; Human Rights Watch 2001). Por tanto, en tratados y análisis posteriores se ha preferido eliminar esta división e incluir una lista homogénea de derechos igualmente exigibles.

Finalmente, en cuanto a las garantías procesales, el ACLAN establece obligaciones relacionadas con el debido proceso y la transparencia de los procedimientos de aplicación del derecho laboral nacional. Sin embargo, no es claro si los niveles de control más estrictos -esto es, los comités de expertos, el arbitraje y las sanciones - se aplican a violaciones de estos derechos procesales por parte de los Estados firmantes (Weiss 2003:711).

\subsubsection{La aplicación del tratado: los órganos, el procedimiento y las sanciones}

Los mecanismos de aplicación del ACLAN pueden ser activados tanto por los gobiernos miembros como por organizaciones de la sociedad civil. En la práctica, todas las quejas por violaciones de los principios del acuerdo un total de 28 hasta abril de 2004-han sido presentadas por ONG, sindicatos y otras organizaciones de la sociedad civil. Esto ha dado lugar a numerosas críticas sobre la inactividad y la falta de voluntad política de los gobiernos para utilizar y desarrollar los mecanismos de control y promoción de derechos estipulados en el acuerdo (Human Rights Watch 2001).

Las quejas se presentan ante la OAN de cualquiera de los tres países miembros. La mayoría de las quejas ha sido presentada ante la OAN de Estados Unidos por violaciones del derecho de asociación sindical por parte del Estado mexicano. Las OAN son órganos estatales nacionales que funcionan como puntos de contacto entre los ministerios de trabajo de los países y el Secretariado de la Comisión para la Cooperación Laboral. En la práctica, las OAN han hecho la mayor parte del trabajo de documentación y de 
estudio de las quejas presentadas. Con base en su análisis, la OAN publican un reporte e intenta una conciliación. Si la conciliación no fructifica, se pasa a la siguiente fase de aplicación, consistente en una revisión del caso a través de una consulta entre los ministerios de trabajo de los tres países. Como se vio anteriormente, este es el máximo nivel de protección para los derechos laborales colectivos. Cuando la queja tiene que ver con estos derechos, como ha sucedido en la mayoría de los casos, la violación da lugar a lo sumo a un reporte y una recomendación conjunta de los tres ministerios.

Sobre el papel, cuando se trate de infracciones de cualquiera de los otros ocho principios protegidos por el ACLAN, si la recomendación ministerial no es implementada, se puede pasar al siguiente nivel de aplicación del acuerdo, esto es, la etapa de evaluación. En esta fase, un Comité de Expertos examina los documentos aportados por los autores de la queja y el gobierno contra la que éste se dirige, y expide un reporte con una conclusión sobre los hechos y una recomendación no obligatoria. Se espera que este reporte sirva como guía para que las partes solucionen la controversia. En la práctica, sin embargo, ninguno de los 28 casos ha pasado de la etapa de consulta ministerial por falta de voluntad política de los gobiernos de los tres países. ${ }^{2}$ En efecto, los casos han terminado o bien con el rechazo de la queja por parte de la OAN o, en la mayoría de las ocasiones, con un débil reporte de la OAN o una recomendación conjunta de los ministerios exhortando a la realización de un seminario o un estudio trinacional sobre el derecho de que trate el caso.

De nuevo sobre el papel, si las partes no pueden conciliar el caso con base en el informe del Comité de Expertos y el derecho involucrado es uno de los tres que acarrean el máximo nivel de protección, el caso puede llegar a la instancia última de aplicación: la resolución de la disputa a través de un panel arbitral. Si se encuentra fundada la acusación, la sanción que el panel puede imponer es una multa al país infractor. El monto de la multa debe ser posteriormente invertido en mejorar la aplicación del derecho violado en el país condenado. Nada obsta, sin embargo, para que dicho país modifique la distribución del presupuesto en la próxima vigencia fiscal y "recupere" la suma pagada mediante una inversión menor en los mecanismos de garantía

\footnotetext{
${ }^{2}$ Una razón adicional que hace que esta etapa sea ineficaz es que cuando la queja sea presentada por uno de los países miembros contra otro, el primero tiene que someterse también al examen de sus prácticas laborales en relación con el derecho por cuya violación ha acusado al otro país. Esto, claramente, desestimula la presentación de quejas por parte de los Estados y el trámite de las quejas más allá de la etapa de la consulta ministerial.
} 
de los derechos laborales, lo que, como lo han señalado varios críticos, dejaría sin efecto el poder disuasivo de la multa (Human Rights Watch 2002). En el muy improbable caso que el país no pague la multa, el país que haya presentado la queja o aquél ante cuya OAN se haya iniciado el procedimiento puede imponer sanciones comerciales equivalentes al monto de la multa.

No obstante, en la práctica ni el mecanismo de arbitraje ni las sanciones pecuniarias o comerciales ha entrado en funcionamiento. Dado que los casos se han quedado en el nivel de estudio y consulta, el análisis de la operación del ACLAN no ofrece elementos de juicio sobre estos aspectos fundamentales de la aplicación de las cláusulas laborales.

\subsubsection{El balance y las lecciones del ACLAN}

Tras diez años de existencia, iqué lecciones ofrece la experiencia de NAFTA con la protección de los derechos laborales? Los estudios sobre el tema son unívocos en resaltar las oportunidades creadas y perdidas por el ACLAN. Entre las primeras se encuentra el establecimiento de un precedente pionero de inclusión de derechos laborales en tratados de libre comercio, que ha servido como base para los lentos avances posteriores que se han logrado en otros tratados que veremos a continuación. Algunos aspectos importantes del acuerdo, como la lista amplia de derechos y la apertura a quejas presentadas por organizaciones de la sociedad civil, son también valiosas lecciones que sirven como modelo para nuevas cláusulas laborales. Otro efecto positivo del ACLAN que ha sido resaltado en la bibliografía es la creación de espacios para la cooperación entre organizaciones de la sociedad civil de los tres países miembros. En efecto, un análisis de los 28 casos muestra que una buena parte de ellos han sido iniciados mediante esfuerzos colaborativos entre ONG de los tres países, especialmente de México y Estados Unidos. Como lo ha mostrado Graubart (2002), este efecto indirecto del ACLAN, que no depende del éxito de los casos o la eficacia de los mecanismos de control del acuerdo, ha sido importante para el surgimiento gradual de redes de cooperación laboral en América del Norte.

Sin embargo, en el balance del ACLAN predominan las lecciones negativas y las oportunidades perdidas. Entre las primeras, es claro que el hecho de que el acuerdo fuese una solución de compromiso lograda a última hora y que los países firmantes no se hubiesen comprometido a niveles de protección más exigentes crearon fallas profundas de diseño institucional en el tratado. Algunas de las más importantes son la multiplicación indefinida de las consultas y los trámites, que, unida a la ausencia de plazos para las diversas etapas, hace que el procedimiento sea muy dilatado e ineficiente. Igualmente importante es la inexistencia de un sistema de resolución de quejas 
independiente de los gobiernos de los países miembros, que asegure la aplicación imparcial y pronta del tratado. Esta falla deja la aplicación de los derechos laborales completamente a la voluntad política (inexistente hasta ahora) de los gobiernos de turno. Otra deficiencia estructural es la ineficaz e innecesaria etapa intermedia de revisión por un Consejo de Expertos. Dada la indefinición de los requisitos para el funcionamiento de éste y la obligación del Estado que presenta la queja de someterse al mismo escrutinio que el Estado acusado, no sorprende que ningún caso haya llegado a esta etapa. Finalmente, otra falla protuberante es la ausencia de mecanismos de control para que, en caso de sanción, la multa no sea recuperada por el Estado castigado mediante una simple redistribución de los recursos fiscales.

Pero incluso con estos vacíos de diseño, el ACLAN podría haber sido mucho más eficaz de lo que hasta ahora ha sido. En este sentido, las oportunidades perdidas son tan notorias como las lecciones negativas que ofrece el texto del tratado. Como lo han señalado numerosos comentaristas, aún el texto existente habría podido ser utilizado por gobiernos proactivos para promover una mejoría gradual de la aplicación de los derechos laborales (Hufbauer y Schott 2002; Elliot y Freeman 2003). En lugar de esto, la realidad del funcionamiento del ACLAN ha mostrado una profunda falta de voluntad política de los gobiernos de los tres países. Esto se debe en parte a que la ausencia de un mecanismo independiente de aplicación del tratado crea un incentivo negativo para la activación del acuerdo por parte de un gobierno contra otro. Dado que todos los países son vulnerables frente a acusaciones de violación de derechos laborales, una acusación probablemente generaría la retaliación del acusado contra el acusador mediante una nueva queja.

Por todas estas razones, en resumen, el ACLAN ha sido justamente exaltado como un precedente importante y una referencia obligada para nuevos intentos de diseñar cláusulas laborales, al mismo tiempo que ha sido duramente criticado por analistas y organizaciones que piden su aplicación estricta y su reforma selectiva (Polaski 2004; Human Rights Watch 2001).

\subsection{El TLC entre Estados Unidos y Jordania}

Aunque Jordania es una economía pequeña y un socio comercial menor de Estados Unidos, el TLC firmado entre los dos países estableció un precedente importante para la protección de los derechos laborales. De hecho, desde que se firmó el tratado en 2000, el debate sobre las cláusulas laborales en Estados Unidos, incluyendo el que llevó a la expedición de la ley TPA por parte del Congreso en 2002, ha estado marcado por la influencia del llamado "modelo jordano" ("Jordan standard"). 
Las novedades del tratado EE.UU-Jordania surgieron en buena medida de las críticas que se habían hecho al ACLAN. Los avances del tratado son cuatro. Primero, las cláusulas laborales están contenidas en el texto mismo del tratado, no en un acuerdo paralelo. Segundo, el tratado establece que las partes "tratarán de asegurar" que los derechos laborales reconocidos internacionalmente sean adoptados y protegidos por el derecho interno. A diferencia del ACLAN, entonces, este tratado incluye como fuentes, además del derecho interno, un propósito general sobre el derecho laboral internacional de la OIT como parámetro al que deben aspirar los ordenamientos nacionales. Tercero, el acuerdo establece expresamente que "es inapropiado promover el comercio a través del debilitamiento del derecho laboral interno". Esta cláusula contra el llamado "dumping social" respondió a las continuas denuncias sobre la llamada "carrera hacia el fondo" en la que los países en desarrollo buscarían competir bajando sus costos laborales mediante la disminución de las protecciones a los trabajadores para atraer la inversión extranjera. Por último, el tratado introdujo una innovación importante en relación con los mecanismos de resolución de disputas, consistente en la paridad del tratamiento de los controversias que surjan en relación con cualquier asunto relativo al tratado, sea de tipo comercial, laboral o ambiental. Por lo tanto, a diferencia del ACLAN, los mismos procedimientos de consulta y resolución de disputas que se aplican a materias comerciales se aplican a asuntos laborales.

Con base en los dos ejes de análisis utilizados para examinar el ACLAN, veamos entonces brevemente qué tipo de modelo crearon estos y otros elementos del tratado EE.UU-Jordania.

\subsubsection{El alcance de los derechos}

A pesar de los avances mencionados, el tratado con Jordania implicó un retroceso en relación con otros aspectos del ACLAN, entre ellos la lista de derechos incluidos. Los derechos protegidos por el tratado son:

1. Condiciones aceptables de trabajo relacionadas con el pago de salario mínimo, horas de trabajo y seguridad industrial;

2. Libertad de asociación;

3. Derecho a la negociación colectiva;

4. Exigencia del establecimiento de una edad mínima para el trabajo infantil;

5. Prohibición del trabajo forzado. 
En comparación con el ACLAN, entonces, fueron excluidas de la lista los importantes derechos a la no discriminación en el empleo por razones de raza, religión, sexo o edad (uno de los derechos fundamentales de la OIT); la igualdad en la remuneración de mujeres y hombres; y la protección de los trabajadores migrantes. Aunque también se excluye el derecho a la huelga, la doctrina uniforme de la OIT ha entendido que éste es inherente a los derechos de asociación sindical y negociación colectiva, que sí están incluidos.

En relación con dos aspectos adicionales del alcance de los derechos, ya mencionados, el modelo jordano contiene avances tímidos pero prometedores. El primero es la inclusión de las normas laborales internacional al menos como fuente de derecho a la que deben aspirar las legislaciones internas. Esto no implica, sin embargo, que en la práctica el acuerdo implique un paso adelante sustancial en relación con la incorporación eficaz del derecho internacional al derecho interno. Dada la vaguedad del lenguaje utilizado las partes sólo deben "tratar de asegurar" la armonía con el derecho laboral internacional-, la obligación de las partes, como en el ACLAN, continúa siendo fundamentalmente aplicar su derecho interno, que están en libertad de modificar. El segundo avance moderado es el nivel mayor de compromiso en relación con los derechos protegidos, mediante la eliminación de la discriminación injustificada del ACLAN entre derechos que gozan de diversos tipos de protección. Sin embargo, como se verá enseguida, la vaguedad de las sanciones hace que en la práctica este modelo de resolución de conflictos pierda gran parte de su efectividad.

Finalmente, el acuerdo guarda silencio en relación con una garantía expresamente incluida en el ACLAN, esto es, las garantías procesales. En este sentido, el modelo jordano es inferior al norteamericano (Weiss 2003).

\subsubsection{Los órganos, el procedimiento y las sanciones}

Gracias a la paridad de tratamiento de las controversias comerciales y laborales, el modelo jordano simplifica notablemente el procedimiento de aplicación de las cláusulas sobre los derechos de los trabajadores. De esta manera, el Comité Conjunto creado para implementar el acuerdo es el organismo competente para examinar y decidir las quejas por violación de las cláusulas laborales (Elliot y Freeman 2003:87).

Sin embargo, la distancia ganada por este rasgo del modelo es perdida por el retroceso que implican dos de sus detalles institucionales. De un lado, a diferencia del ACLAN, las quejas pueden ser presentadas sólo por los gobiernos. De esta forma, se cierra la puerta a la participación de organizacio- 
nes de la sociedad civil, que ha demostrado ser el único mecanismo eficaz para la utilización y el desarrollo del ACLAN. De otro lado, las sanciones por incumplimiento son vagas. Cuando el Comité Conjunto no logre dirimir una disputa, la parte que interpuso la queja puede "tomar cualquier acción apropiada y proporcional" en material comercial para castigar la conducta infractora. El lenguaje es tan vago que o bien puede dar lugar al abuso del poder de sanción o bien, como es más probable, a su inaplicación.

\subsubsection{El balance del modelo jordano}

Cuando se examina con detenimiento el texto del tratado entre Estados Unidos y Jordania -que, como vimos, no aumenta el nivel de protección laboral en la práctica - es difícil entender el furor contra su aprobación que se suscitó en los círculos republicanos dentro del Congreso de Estados Unidos y entre los economistas opuestos a la vinculación del comercio y los derechos laborales. Aunque el modelo jordano implica algunas innovaciones importantes, en varios aspectos es menos protector que el ACLAN.

Quizás la fuerte oposición de los republicanos y los economistas más entusiastas del libre comercio sin condiciones se deba no tanto a los efectos directos del tratado, sino al potencial que tienen algunas de sus cláusulas de servir de precendentes para otros tratados que pueden desarrolladas y convertirlas en formas de protección más exigentes de los estándares laborales. Este es el caso, por ejemplo, de las normas sobre paridad de tratamiento de asuntos comerciales y laborales, que, si van acompañada de compromisos y sanciones claras, pueden dar lugar a la simplificación del procedimiento que es necesaria para la aplicación eficiente de las cláusulas laborales. Este potencial daría cuenta, por ejemplo, de la apasionada crítica de Bhagwati contra el tratado en el Congreso de Estados Unidos y la posibilidad de que el "modelo jordano" fuera utilizado en tratados posteriores (Bhagwati 2001).

En resumen, el tratado EE.UU-Jordania por sí mismo no implicó avances importantes en la protección de los derechos laborales, pero abrió la puerta a la experimentación con mecanismos más ágiles y eficaces de implementación. Como se verá en la sección de recomendaciones de este escrito, algunas variaciones del modelo jordano, unidas a elementos valiosos del ACLAN, pueden constituir una cláusula laboral equilibrada y eficaz.

\subsection{El TLC entre Estados Unidos y Chile}

Un antecedente cercano e importante para Colombia es la negociación del TLC de Chile con Estados Unidos. Como el TLC colombiano, la nego- 
ciación del chileno tuvo lugar con base en la autoridad dada al gobierno estadounidense por el TPA de 2002. De acuerdo con los lineamientos del TPA, el acuerdo incluye cláusulas laborales, tomadas fundamentalmente del modelo jordano. Cláusulas casi idénticas a las del modelo chileno fueron introducidas en el TLC entre Estados Unidos y Singapur, que fue negociado y ratificado al mismo tiempo que el chileno.

\subsubsection{El alcance de los derechos}

El tratado EE.UU-Chile implica los mismos avances y retrocesos del modelo jordano en relación con el ACLAN. Al igual que en el caso de Jordania, las estipulaciones laborales hacen parte del texto mismo del tratado. La lista de derechos es la misma que la del modelo jordano. Esto implica que se excluyen los importantes derechos a la no discriminación, la equidad de pago entre hombres y mujeres y la protección de los migrantes. El sistema de fuentes es el mismo que el del tratado EE.UU-Jordania: a pesar de que se hace una referencia a la armonización del derecho interno con el derecho laboral internacional, se limita la exigibilidad a la aplicación del derecho nacional. Igualmente, se mantiene la eliminación de los niveles de protección, de tal forma que todos los derechos incluidos son objeto del mismo mecanismo de resolución de controversias. Finalmente, un avance en relación con el modelo jordano es la inclusión de garantías procesales, esto es, al acceso a una justicia laboral justa, equitativa y transparente (USTR 2004).

\subsubsection{Los órganos, el procedimiento y las sanciones}

El tratado crea un Consejo Chileno-Estadounidense para Asuntos Laborales, encargado de vigilar la implementación de las cláusulas laborales, coordinar las consultas relativas a ellas y organizar actividades de cooperación en esta materia. Una innovación interesante es la creación de un órgano de cooperación continua para impulsar el cumplimiento de los derechos laborales fundamentales de la OIT.

Los mecanismos coercitivos son similares a los del modelo jordano. La falla más protuberante es de nuevo la clausura del sistema de quejas a la participación de partes distintas de los dos gobiernos. Los casos laborales pueden surgir sólo a partir de quejas presentadas por uno de los dos gobiernos contra el otro. Como se vio en el análisis del ACLAN, es altamente improbable que se presente este tipo de quejas, de tal forma que la exclusión de la participación de las sociedades de los dos países en la iniciación de los casos es una falla que, en la práctica, implica la ineficacia de las cláusulas laborales. 
El procedimiento para solucionar estas improbables controversias comienza con consultas cooperativas entre las partes para resolver la queja de una de ellas. Si después de 60 días no se ha llegado a un acuerdo, la parte que inició el procedimiento puede llevar el caso al mismo órgano de resolución de controversias que conoce de los casos comerciales. ${ }^{3}$ La sanción prevista en caso de condena por parte del panel arbitral es una multa, que no puede exceder US \$15 millones al año. Si la multa no es cancelada, la parte demandante puede imponer sanciones comerciales consistentes en aranceles proporcionales a la multa impuesta.

\subsubsection{El balance del tratado EE.UU-Chile}

El tratado chileno no contiene avances importantes frente a los modelos norteamericano y jordano. Con excepción de la reincoporación de los derechos laborales procesales y la creación de un organismo de cooperación (cuya financiación es incierta), presentes en el ACLAN pero no en el modelo jordano, no aporta elementos nuevos frente a su antecesor inmediato. Por el contrario, continúa algunos rasgos de éste que implican en la práctica la ineficacia de las cláusulas laborales, especialmente la imposibilidad de que ONG, sindicatos y otras organizaciones de la sociedad civil presenten quejas. La tendencia continuada por el tratado chileno, entonces, apunta a un sistema excluyente donde los asuntos laborales son del dominio de los gobiernos y están clausurados a la participación de las sociedades de los países firmantes.

\subsection{Las disposiciones laborales del CAFTA}

Aunque no ha sido ratificado por los órganos legislativos de Estados Unidos y los países centroamericanos que lo firmaron (Costa Rica, El Salvador, Honduras, Guatemala y Panamá) y, por tanto, no constituye un precedente en firme, el CAFTA (US-Central American Free Trade Agreement) ofrece algunas lecciones para el TLC colombiano que vale la pena señalar por lo menos brevemente.

Las lecciones tienen que ver no tanto con el contenido de las cláusulas laborales -que reproducen casi en su totalidad los modelos jordano y chileno-, sino con las consecuencias nocivas que estos modelos tienen tanto

\footnotetext{
${ }^{3}$ La única excepción a esta regla son los casos sobre quejas relativas a violaciones de derechos laborales que tuvieron lugar en sectores de exportación. En estos casos, un panel especial creado para el efecto resolverá el conflicto (USTR 2004).
} 
para la protección de derechos laborales en contextos violentos como el centroamericano y el colombiano como para las posibilidades de aprobación que un TLC de este tipo tiene en el Congreso norteamericano. En cuanto a lo primero, las insuficiencias anotadas en relación con el modelo chileno se agravan profundamente en un contexto que, como el centroamericano o el colombiano, está marcado por graves violaciones de los derechos humanos, incluyendo la violencia física contra miembros y líderes de organizaciones sindicales. Por ejemplo, la inexistencia de mecanismos de presentación de quejas por parte de la sociedad civil y la falta de garantías para la eficacia y la adecuada financiación del organismo bilateral de cooperación laboral han profundizado las dudas sobre la continuación -o incluso el agravamientode las múltiples violaciones de los derechos laborales en Centroamérica documentados por la OIT y ONG locales e internacionales. Esto explica en parte la actual controversia en el Congreso de los Estados Unidos en relación con la aprobación del CAFTA, en la cual los informes de violaciones sistemáticas de los derechos humanos de los trabajadores han sido un factor importante (Human Rights Watch 2004).

El impasse actual del CAFTA constituye, entonces, una advertencia sobre la necesidad de que países como Colombia, Guatemala o El Salvador que tienen algunas de las trayectorias más negativas en el mundo en relación con la protección de los derechos laborales fundamentales básicos como el trabajo infantil y la asociación sindical- incluyan en sus TLC cláusulas explícitas y eficaces de protección de sus derechos laborales. La inclusión de este tipo de cláusula, cuyos lineamientos se ofrecen en la siguiente sección, es necesaria en vista de los debates y críticas justificadas que surgirán en el Congreso de los Estados Unidos en relación con el negro historial colombiano en materia de garantía de los derechos humanos de los trabajadores.

4. Conclusión: Recomendaciones sobre el contenido de la cláusula laboral en el TLC entre Colombia y Estados Unidos

Con fundamento en el análisis y las razones ofrecidos en las secciones anteriores, la cláusula laboral del TLC debe tener las siguientes características:

\subsection{Lista de derechos: la lista debe ser tomada del ACLAN y, por tanto, debe incluir los siguientes principios:}

1. Libertad de asociación y derecho de asociación sindical;

2. Derecho de negociación colectiva;

3. Derecho a la huelga;

4. Prohibición de trabajo forzado; 
5. Protecciones laborales para niños y jóvenes;

6. Garantía de derechos laborales mínimos;

7. Eliminación de la discriminación en el trabajo;

8. Igual remuneración para mujeres y hombres;

9. Prevención de lesiones y enfermedades profesionales;

10. Compensación en caso de lesiones o enfermedades profesionales; y

11. Protección para trabajadores migrantes.

4.2. Fuentes de derecho: cada una de las partes debe compromenterse a aplicar tanto su derecho nacional como las convenciones de la OIT que haya suscrito, interpretadas de acuerdo con los lineamientos de la OIT. Dado que Colombia ha ratificado todas las convenciones fundamentales de la OIT y que Estados Unidos no ha suscrito varias de ellas, esta solución es a la vez lo suficientemente flexible como para comprender los dos casos y lo suficientemente exigente como para establecer un parámetro internacional adecuado y ampliamente reconocido.

4.3. Derechos procesales: como en el ACLAN y el TLC entre Estados Unidos y Chile, las partes se deben comprometer a garantizar el acceso a una justicia equitativa, transparente y rápida que haga eficaz los derechos sustantivos que se comprometen a proteger.

4.4. Presentación de quejas: los mecanismos de resolución de controversias deben poder ser activados mediante quejas tanto de uno de los Estados miembro como de terceras partes (empresas, ONG, sindicatos, organizaciones de la sociedad civil, etc.). Se debe incluir, entonces, el fundamental mecanismo de las quejas de terceros, sin el cual, según se vio anteriormente, las cláusulas laborales son ineficaces.

4.5. Mecanismo de resolución de controversias: las quejas sobre cualquiera de los derechos laborales incluidos en el tratado, tanto los sustanciales como los procesales, deben ser tramitadas por un organismo independiente e imparcial. Para evitar la politización de los páneles bilaterales evidente en los tratados analizados anteriormente, este organismo debe tener competencia para investigar, promover consultas y conciliaciones, dictar laudos e imponer sanciones en los casos que corresponda. En cualquier caso, el tratamiento de las controversias laborales debe ser por lo menos equivalente -en celeridad, transparencia y efectividad - al que el TLC establezca para los conflictos comerciales.

4.6. Procedimiento: las quejas de las Estados miembros o de terceras partes deben ser presentadas directamente ante el organismo independiente delineado en el párrafo anterior. El procedimiento debe ser el mismo para las quejas sobre cual- 
quier derecho sustancial o procesal. El TLC debe establecer plazos perentorios y ágiles para la tramitación de la presentación de pruebas, la fase de conciliación y consulta con los gobiernos, y el laudo arbitral.

4.7. Sanciones: cuando el organismo independiente de resolución de controversias encuentre fundada la queja y no se logre una conciliación, podrá imponer una multa al Estado infractor. Como en el CAFTA, la multa deberá ser invertida posteriormente en programas gubernamentales de mejoramiento de la aplicación del derecho violado. A diferencia del CAFTA, dichos programas deben aprobados por el organismo independiente de resolución de conflictos. Además, el Estado infractor deberá demostrar que el monto de la multa no será deducido de otros rubros fiscales dedicados a la protección de derechos laborales.

En caso de renuencia del Estado infractor a pagar y ejecutar la multa en estos términos, la otra parte impondrá sanciones comerciales sólo por el monto de la multa y sólo sobre los productos provenientes del sector donde se produjo la violación de los derechos laborales.

4.8. Cooperación bilateral: como en los tratados previos firmados por Estados Unidos, se deberá constituir un organismo bilateral de análisis y cooperación técnica para promover el cumplimiento de los derechos laborales en los países miembros. Para evitar los problemas de financiación e ineficacia de estos órganos en acuerdos similares, el TLC debe especificar un monto mínimo que cada una de las partes debe aportar para financiar sus operaciones. Este organismo de cooperación bilateral deberá trabajar en colaboración con la OIT en programas generales de evaluación y mejoramiento del cumplimiento de los derechos laborales.

\section{Referencias Bibliográficas}

Alston, Philip. 1996. "Labor Rights Provisions in U.S. Trade Law: 'Aggressive Unilateralism'?", en Lance Compa y Stephen Diamond, eds, Human Rights, Labor Rights, and International Trade. Philadelphia: University of Pennsylvania Press, pp. 71-98.

Audley, John, Demetrious Papademtriou, Sandra Polanski y Scott Vaughan. 2003. La Promesa y la Realidad del TLCAN. Lecciones de México para el Hemisferio. Nueva York: Carnegie Endowment for International Peace.

Baker, Dean, Gerald Epstein y Robert Pollin, eds. 1998. Globalization and Progressive Economic Policy. Cambridge: Cambridge University Press.

Banco Mundial. 2003. "Company Codes of Conduct and International Standards: An Analytical Comparison" (Octubre).

Bhagwati, Jagdish. 1999. "Third World Intellectuals and NGOs-Statement against Linkage" (http://www.columbia.edu/ jb38, mayo de 2004) 
- 2001. "The Jordan Free Trade Agreement: The Wrong Template." Testimonio ante el Comité Financiero del Senado de Estados Unidos. (www.columbia.edu/ jb38, mayo de 2004)

- 2004. In Defense of Globalization. Oxford: Oxford University Press.

Bonacich, Edna y Richard Appelbaum. 2000. Behind the Label. Inequality in the Los Angeles Apparel Industry. Berkeley: University of California Press.

Diamond, Stephen. 1996. "Labor Rights in the Global Economy: A Case Study of the North American Free Trade Agreement", en Lance Compa y Stephen Diamond, eds, Human Rights, Labor Rights, and International Trade. Philadelphia: University of Pennsylvania Press, pp. 199-226.

Dicken, Peter. 1998. Global Shift: Transforming the World Economy. Nueva York: Guilford.

Dreier, Peter and Richard Appelbaum. 1999. "The Campus AntiSweatshop Movement." The American Prospect 10, pp. 10-24.

Elliot, Kimberly. 2003. "Labor Standards and the Free Trade Area of the Americas." Working Paper, Institute for International Economics.

Elliot, Kimberly y Richard Freeman. 2003. Can Labor Standards Improve under Globalization? Washington: Institute for International Economics.

Fung, Archon, Dara O'Rourke y Charles Sabel. 2001. Can We Put an End to Sweatshops? Boston: Beacon Press.

Garay, Luis Jorge. 2002. "Estándares laborales y comercio: Una aproximación a la problemática." Cuadernos de Economía, no. 37, pp. 109-138.

Gereffi, Gary, Miguel Korzeniewicz, y Roberto Korzeniewicz. 1994. "Introduction: Global Commodity Chains", en Gary Gereffi y Miguel Korzeniewicz, eds. Commodity Chains and Global Capitalism. Westport: Praeger, pp. 1-16.

Gereffi, Gary, David Spener y Jennifer Bair, eds. 2002. Free Trade and Uneven Development. The North American Apparel Industry after NAFTA. Philadelphia: Temple University Press.

Graubart, Jonathan. 2002. "The Intersection of Transnational Activism and Soft Law: How Activists Exploit NAFTA's Labor and Environmental Accords". Ph.D. dissertation. University of Wisconsin-Madison.

International Labor Rights Fund. 2004. "Comments Concerning Proposed United States-Andean Free Trade Agreement" (presentado al Congreso de Estados Unidos por Jeffrey Vogt, Assistant General Counsel).

Hufbauer, Gary y Jeffrey Schott. 2002. "North American Labor Under Nafta." Working Paper, Institute for International Economics.

Human Rights Watch. 2001. Trading Away Rights: The Unfulfilled Promise of NAFTA's Labor Side Agreement. Nueva York: Human Rights Watch.

-2002. "Labor Rights and Trade: Guidance for the United States in Trade Accord Negotiations." (Octubre). 
-2004. "CAFTA's Weak Labor Protections: Why the Present Accord Should be Opposed" (Marzo)

Jessop, Bob. 2002. The Future of the Capitalist State. Cambridge: Polity Press.

Keck, Margaret y Kathryn Sikkink. 1998. Activists Beyond Borders. Advocacy Networks in International Politics. Ithaca: Cornell University Press.

MacEwan, Arthur. 2001. Neo-Liberalism or Democracy? Economic Strategy, Markets, and Alternatives for the $21^{\text {st }}$ Century. Londres: Zed.

Moran, Theodore. 2002. Beyond Sweatshops: Foreign Direct Investment and Globalization in Developing Countries. Washington, D.C.: Brookings.

Polaski, Sandra. 2004. "Labor in North American Integration: Issues, Interests and Actors". Trabajo presentado en el Congreso "Integración Norteamericana: Migración, Comercio y Seguridad". Ottawa, abril.

Reina, Mauricio. 2004. "Como Burro Amarrado", El Colombiano, 17 de abril.

Rodríguez Garavito, César A. 2005a Globalización, Gobernanza y Derechos Laborales. Bogotá y México: ILSA y CIDE.

-2005b. "Global Governance, Cross-Border Organizing and Labor Rights: Codes of Conduct and Anti-Sweatshop Struggles in Global Apparel Factories in Mexico and Guatemala." Politics $\mathcal{E}$ Society, vol. 33, no. 1.

-2005c. "Nike's Law: Anti-Sweatshop Coalitions, Transnational Corporations and the Struggle over International Labor Rights in the Americas", en Boaventura de Sousa Santos y César A. Rodríguez Garavito, eds. Law and Counter-Hegemonic Globalization: Toward a Subaltern Cosmopolitan Legality. Cambridge: Cambridge University Press.

Ross, Andrew. ed. 1997. No Sweat. Fashion, Free Trade and the Rights of Garment Workers. New York and London: Verso

Sassen, Saskia. 1998. Globalization and Its Discontents. New York: The New Press.

Sachs, Jeffrey (1999). Globalization and The Rule of Law. New Haven: Yale University Press.

USTR (Unites States Trade Representative). 2004. "Summary of U.S.Chile FTA Labor Chapter" (www.ustr.gov/new/fta/Chile/summaries, Mayo de 2004)

Weiss, Marley. 2003. "Two Steps Forward, One Step Back - or Vice Versa: Labor Rights under Free Trade Agreements from NAFTA, through Jordan, via Chile, to Latin America, and Beyond." University of San Francisco Law Review, vol. 37, pp. 689-755. 